\title{
The detection of length and orientation changes in dynamic vibrotactile patterns
}

\author{
SANDRA L. SCHNEIDER, BARRY HUGHES, WILLIAM EPSTEIN, and PAUL BACH-Y-RITA \\ University of Wisconsin, Madison, Wisconsin
}

\begin{abstract}
Vibrotactile thresholds for the detection of length and orientation changes were determined for dynamic line patterns presented on the Optacon in a forced-choice procedure. Even the smallest changes in both length $(1.2 \mathrm{~mm})$ and orientation $\left(7^{\circ}\right)$ could be detected in the absence of any other changes in the vibrotactile pattern. The introduction of translations increased the length-change threshold to approximately $4.0 \mathrm{~mm}$, and the introduction of length changes increased the orientation-change threshold to approximately $25^{\circ}$. The effects of several additional variables were assessed; these included the use of filled and unfilled lines, the timing of presentation, and the position of stimuli on the array. The implications for the perception of depth in dynamic vibrotactile patterns are discussed.
\end{abstract}

Much of the current interest in vibrotactile perception has developed in response to the need for sensory substitution systems for the blind (Bach-y-Rita, 1972; Bliss, Katcher, Rogers, \& Shepard, 1970). This focus on vibrotactile perception rests on the implicit assumption that some of the important information acquired through visual stimulation can also be acquired through vibrotactile stimulation. Although clinical investigations (e.g., Bach-y-Rita, 1972) suggest that this assumption is not unreasonable, systematic investigations are needed to determine the extent to which information for visual perception can be transformed into information for vibrotactile perception.

Many previous investigations of vibrotactile perception, particularly with a device called the Optacon, have focused on factors presumed to affect the perception of static and scanned two-dimensional patterns, especially letters (Craig, 1976, 1980, 1981, 1983a, 1983b; Craig \& Sherrick, 1982; Loomis, 1974, 1980, 1981). This work, as well as that of Kirman $(1975,1983)$ on vibrotactile apparent motion, has been concerned with the spatial acuity and temporal integration of vibrotactile stimulation, particularly as it contrasts with that of vision and audition. Although these investigations serve a valuable function in the analysis of vibrotactile perception, there are other types of information potentially available in vibrotactile stimulation. To the extent that the sense of sight derives its value from its capability to provide information about depth relations in the environment, the success of vibrotactile perception as a substitute for vision can be measured in terms of the number and kind of spatiotemporal invari-

This research was supported by the National Science Foundation Grant ECE-841-0840 awarded to Paul Bach-y-Rita. We gratefully acknowledge the assistance of Jon Birschbach, Jean Chapman, Willard Thurlow, Fred Wightman, and two anonymous reviewers.

The authors' mailing address is: Department of Psychology, W. J. Brogden Psychology Building, University of Wisconsin, 1202 W. Johnson St., Madison, WI 53706. ants related to depth that it can make available to the perceiver (Epstein, 1985; Kirman, 1973).

Certain types of movement, or systematic changes constituting spatiotemporal invariants, provide visual information for the perception of depth. As Wallach and O'Connell (1953) pointed out in their demonstration of the kinetic depth effect, two important variables for the perception of movement in depth are systematic changes in the length and orientation of retinal contours. The purpose of the present investigation was to determine the thresholds for the detection of changes in length and in orientation of lines from dynamic presentations of vibrotactile stimulation. If these changes can be reliably detected, the potential for the vibrotactile perception of movement in depth can be explored within the limits of vibrotactile sensitivity.

\section{EXPERIMENT 1 Detection of Length Changes}

The first experiment examined the ability to detect changes in the length of a line presented as a dynamic vibrotactile pattern. Length-change thresholds were derived for dynamic patterns composed of filled lines as well as dynamic patterns represented only by the endpoints of lines. Length-change thresholds were also examined under conditions in which dynamic patterns involved translations of lines in addition to length changes.

\section{Method}

Subjects. The subjects were 4 female and 2 male sighted introductory psychology students who elected to participate either for course credit or for payment of $\$ 4$ per hour. No subject had previously participated in an experiment concerning vibrotactile psychophysics or had had prior experience using the Optacon.

Apparatus. Vibrotactile stimulation was presented to the user's left index fingertip via an Optacon (Telesensory Systems, Inc.), a device originally intended as a reading aid for the blind. The Optacon has a 6 column $\times 24$ row array of piezoelectric-driven vibrotactile pins. The columns, which lie parallel to the longitudi- 
nal axis of the finger, are separated by approximately $2.4 \mathrm{~mm}$, and the rows are separated by approximately $1.2 \mathrm{~mm}$. The vibrotactile pins, or tactors, become activated in response to the activation of a corresponding portion of a 6 column $\times 24$ row array of photosensitive elements. Typically this activation occurs in response to the presence of light in the optical field of the Optacon camera. The transformed pattern of light is experienced as a pattern of vibrations, occurring at approximately $230 \mathrm{~Hz}$.

For the present experiment, the Optacon camera was removed and the Optacon was interfaced with an IBM-PC, which generated the patterns of stimulation. The custom interface is capable of transmitting representations of a $6 \times 24$ array directly to the vibrotactile array in frames with discrete steps at rates that are multiples of a 4.35-msec vibration cycle time. This transmission ability, along with the appropriate software, enables the presentation of a virtually unlimited number of patterns of vibrotactile stimulation. Be- cause fingertip contact tends to be poor along the bottommost six rows of the Optacon array, the presentation of vibrotactile patterns was limited to the $6 \times 18$ array resulting when these rows are omitted from the original array.

A five-button response panel was interfaced with the IBM-PC for the collection and storage of data. The two buttons to be used for responses were clearly labeled. Earphones connected to a whitenoise generator were used to mask the reverberations produced by the Optacon in response to vibrations of the pins.

Stimuli. The basic stimuli were dynamic patterns of vibrotactile lines presented lengthwise on the Optacon array. These dynamic patterns were created by presenting one component line for a 435 msec stimulus duration (D) followed by a 435 -msec interstimulus interval (ISI), then a second component line for a 435-msec D followed by another 435 -msec ISI. This series was presented three times in succession, resulting in a recurring cycle of change which,
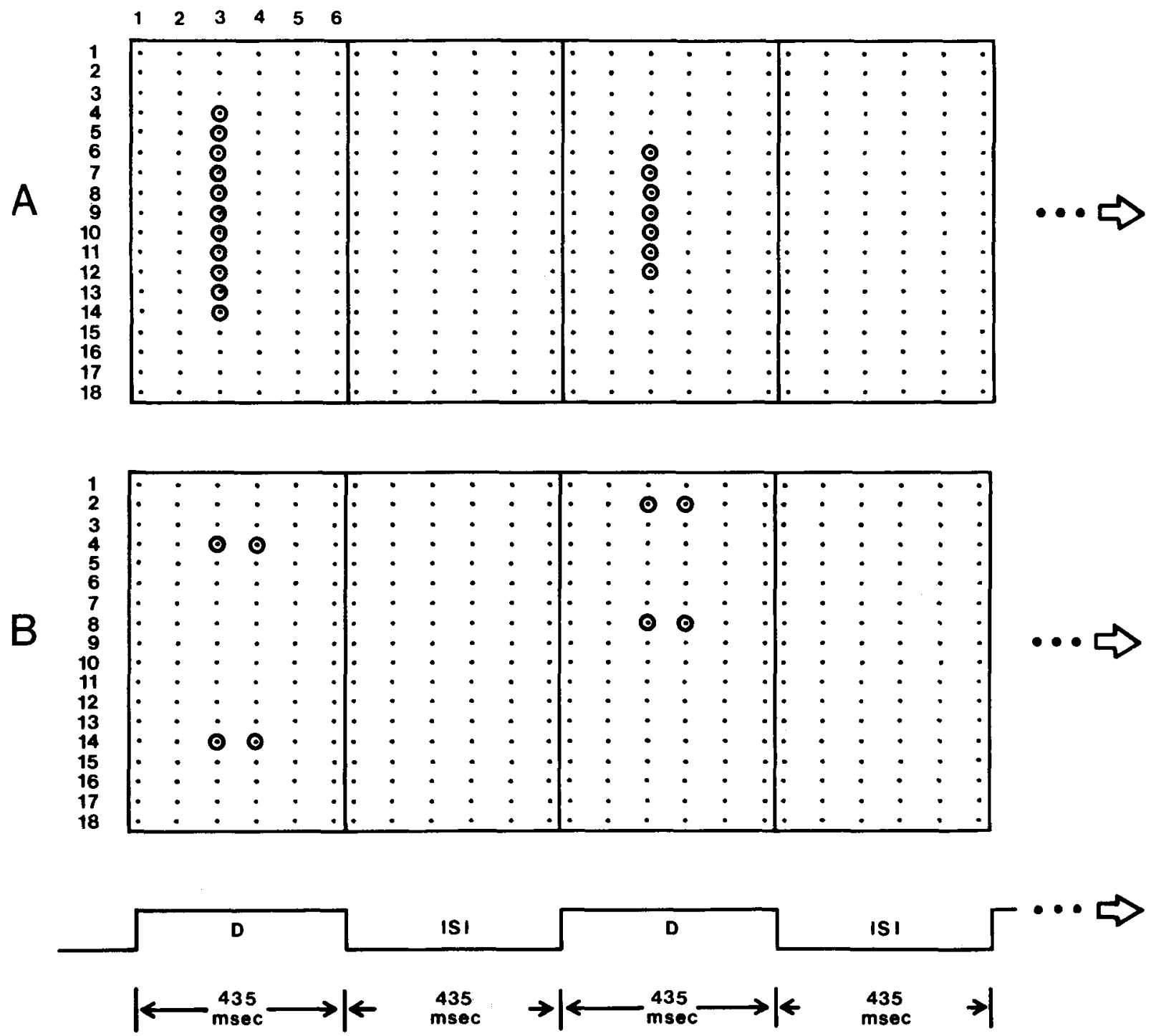

Figure 1. Examples of one of the three cycles in a vibrotactile line event. Each cycle consists of a first component line with a 435-msec $D$ followed by a 435 -msec ISI, then a second component line with a 435-msec D followed by a 435-msec ISI. Activated tactors within the sequence are circled. (A) An example that depicts a line in the filled condition with a 4-vtu length change. (B) An example that depicts a line in the unfilled condition with a 4-vtu length change and a 4-vtu translation toward the top of the array. 
for present purposes, is referred to as an event. The Ds and ISIs were selected to eliminate the possibility that masking would affect thresholds. As Craig (1976) has demonstrated in investigations of letter recognition, masking affects discrimination whenever patterns are presented within 0 and 300 to 400 msec of each other.

The length of the first component line in all events was held constant at 10 vibrotactile units (vtu, approximately $1.2 \mathrm{~mm}$ each). Although the second component line had the same centerpoint as the first, its length was determined by the magnitude of the length change specified for the particular event. Figure 1A depicts the first of the three cycles comprising an event wih a 4-vtu length change. Note that the line first becomes four units shorter (as pictured), then becomes four units longer as it returns to the original length. Thus, changes of length within each event comprised both a shortening and a lengthening, which recurred in a cyclic dynamic pattern.

The lines within each event were either filled or unfilled. In the filled condition, each tactor from one end of the line to the other vibrated during presentation, as illustrated in Figure $1 \mathrm{~A}$. In the unfilled condition, only the endpoints of the line vibrated during presentation, as illustrated in Figure 1B. To make these endpoints more discriminable and to diminish overall differences in intensity across conditions, each endpoint in the unfilled lines was represented by two vibrating pins in adjacent columns of the Optacon array.

In the absence of any additional manipulations of the line events, reports of changing length could be based solely on detection of movement during the event rather than on actual perceptions of length change. To rule out this possibility, the variable referred to as translation was introduced. In addition to variations in length, the centerpoint of the second component line in each event was translated by $0,2,4$, or 6 vtu toward the top or bottom of the array. Hence, movement during the event could represent a translation, a change in length, or both. Figure 1B depicts the first cycle of an event in the unfilled condition with a 4-vtu length change and a 4-vtu translation toward the top of the array.

Several secondary manipulations were included in the presentation of each event to determine whether the location of vibrotactile stimulation on the fingertip affected discriminability of length change. (1) The column position of the lines was varied, each event occurring on the left side of the array (columns 1-2), in the middle (columns 3-4), or on the right side of the array (columns 5-6). (2) The starting position of the line was varied, each event beginning at the top of the array (rows 1-10), in the middle (rows 4-14), or at the bottom of the array (rows 8-18). (3) Translation direction was either toward the distal portion of the array (up) or toward the proximal portion of the array (down). (4) The length sequence comprised an increase in line length followed by a decrease or a decrease in line length followed by an increase.

Design and Procedure. An adaptive psychophysical technique, a transformed up-down method, was used to determine lengthchange thresholds in a forced-choice procedure. (For a complete discussion of this method, see Levitt, 1971, or Penner, 1978.) On each trial, two events were presented in succession. In one event, a change in length occurred; in the other, there was no change in length. The subject's task was to press the response button indicating in which event, the first or second, the length change had occurred. The length change was increased by 1 vtu following one incorrect response and decreased by 1 vtu following two correct responses. In this way, trials converged on the level at which the probability of a correct response was 0.707 , a close approximation to the 0.75 level adopted as the length-change threshold.

The experiment was presented in 64-trial blocks. Each block began with a 5-vtu change in length. The changes in length presented

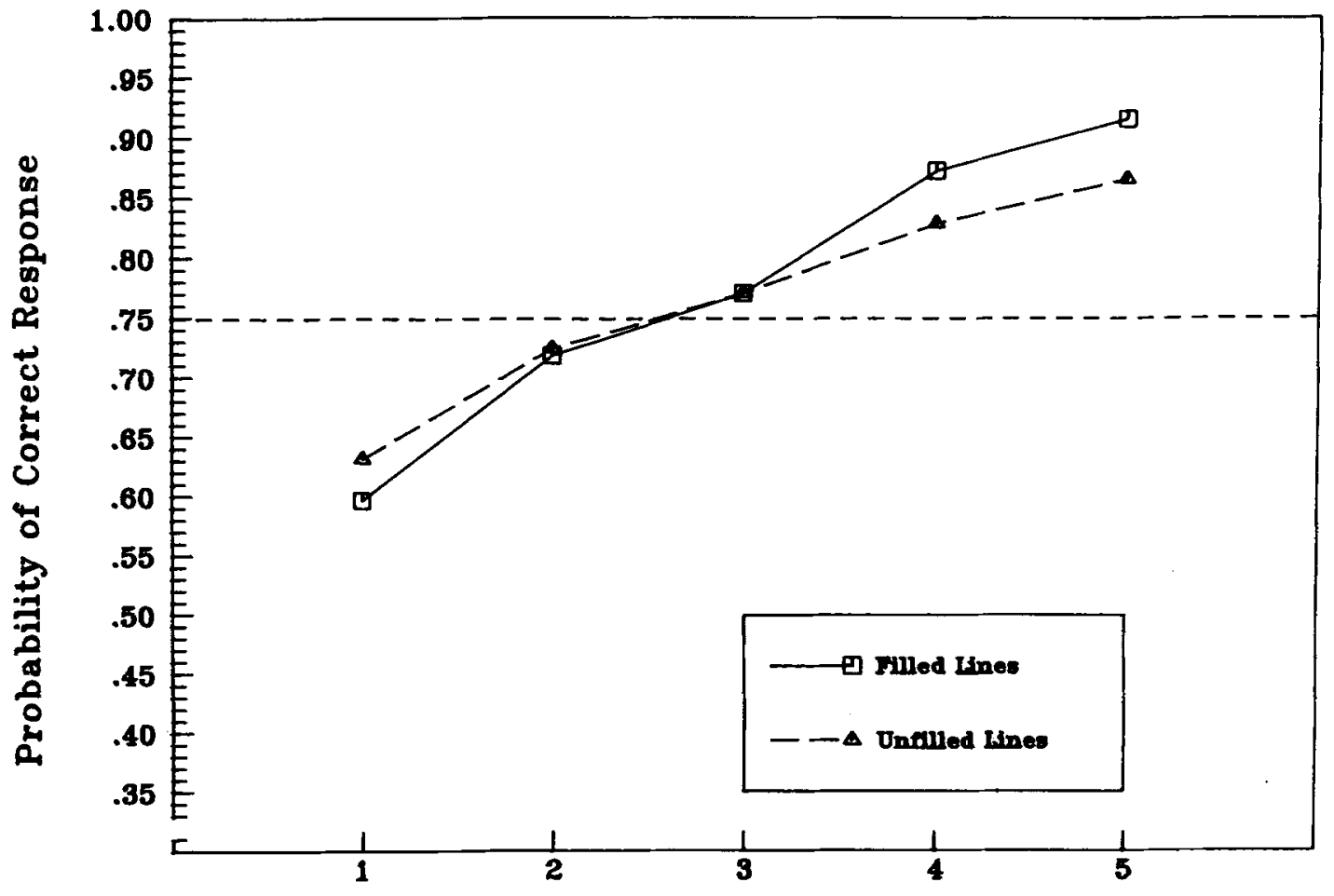

Change in Length (vtu)

Figure 2. The probability of correct identification of length-change events for the filled- and unfilled-line conditions. The dashed line represents the 0.75 value adopted as threshold. 
thereafter were determined by the correctness of the subject's responses with lower and upper limits of 1 and 6 ver, respectively. ${ }^{1}$ The translation manipulation was counterbalanced within each block, but the level of translation remained the same for both events on any given trial. Whether the length change cocursed in the first or secand event was detemines rasdomly for eacis tial. The leveis of the socondary variables of column position, slarting position, translation direction, and length sequence were also determined randomly but, again, the levels remained the same for both events on a given trial.

In each session, two to three blocks of trials were completed in either the filled-or unfilled-line condition. Half of the subjects were chosen randomly to begin their first session with the filled-line condition; the remaining subjects began with the unfilled condition. The line conditions were alternated for each session thereafter. Each subject completed as many sessions as necessary to have at least 100 trials on either side of the 0.75 threshold for the filled condition and for the unfilled condition.

The subjects were reminded at the beginning of each session to ignore translations of the line and to attend only to changes in length. After each response, the next trial was presented automatically until the block was completed. The subjects were encouraged to rest between blocks and to complete only two blocks in the session if they were fatigued.

\section{Results}

As illustrated in Figure 2, the threshold for detecting changes in length is less than 3 vtu for both filled and unfilled dynamic line patterns. However, the abiliry to detect changes in length is not significantly different for the filled and unfilled conditjons $(F<1)$. This finding indicates that resolution difficulties encountered in the presentation of dynamic filled-line patterns on the Optacon can be eliminated without affecting discriminations of length changes by presenting line patterns consisting only of endpoints.

The ability to detect changes in length, however, is significantly affected by concurrent translations of the vibrotactile lines $[F(3,5)=21.34, p<.001]$. Figure 3 presents length-change thresholds (pooled over the filled and unfilled conditions) as a function of translation. When there is no translation, the threshold for length-change detection is $1 \mathrm{vtu}(1.2 \mathrm{~mm})$, whereas in the presence of a 2-, 4-, or 6-vtu translation, the length-change threshold is slightly above 3 vtu. Hence, even the smallest changes

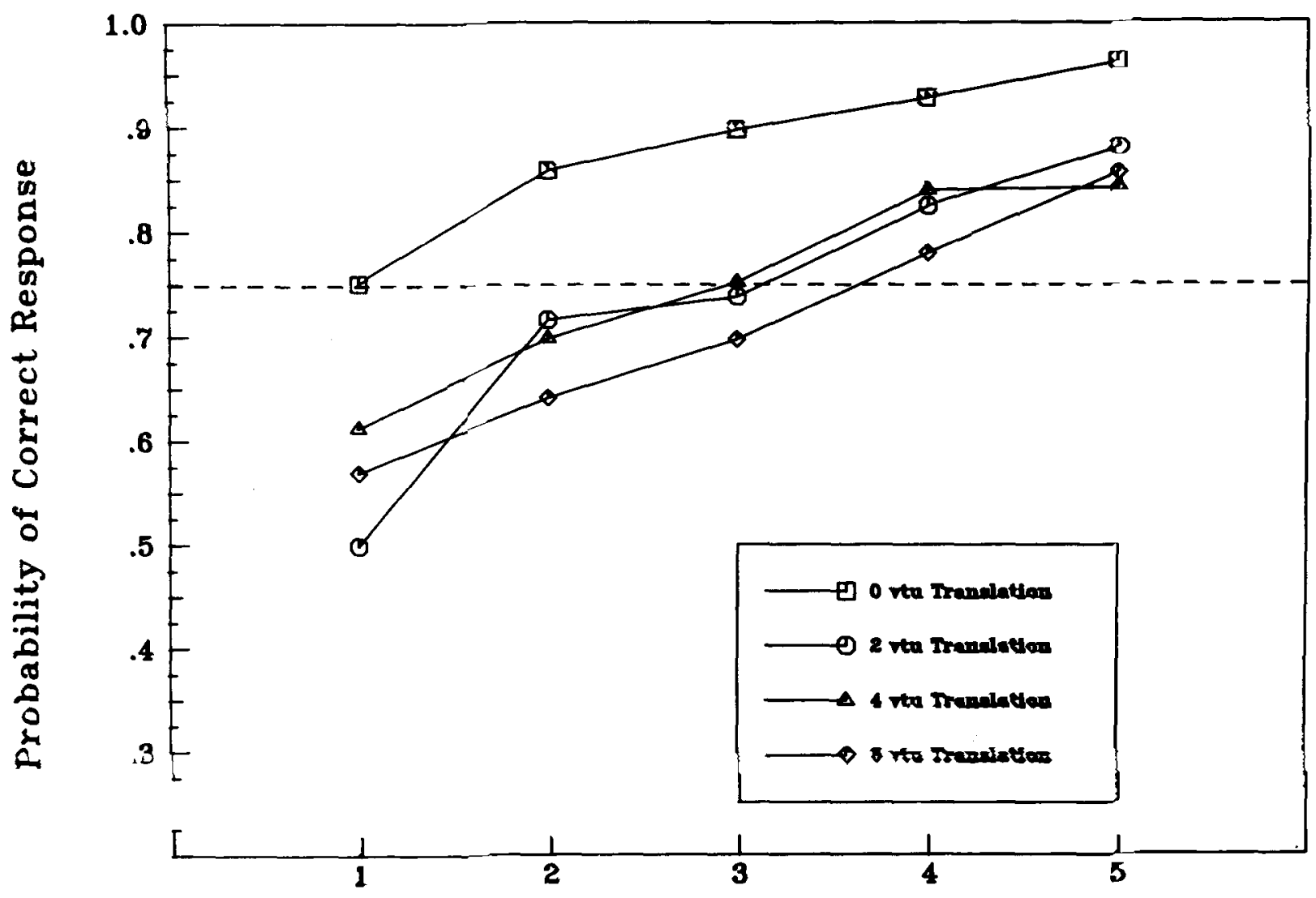

\section{Change in Length ( $v t u)$}

Figure 3. The probability of correct identification of length-change events for $0-, 2-, 4$, and 6-vtu translations. The dashed line represents the 0.75 value adopted as threshold. 
in length can be detected in the absence of translations. Although the presence of a translation significantly reduces the ability to detect length changes $[F(1,5)=$ $65.00, p<.001$, by pairwise comparison], the magnitude of the translation $(2,4$, or $6 \mathrm{vtu})$ does not have a significant impact.

The ability to detect changes in length is not dependent on the location of stimulation within the $6 \times 18$ array. None of the secondary variables, including column position, starting position, translation direction, and length sequence, has a significant effect on length-change discrimination. Neither does the appearance of the change in length in the first or second event affect performance.

\section{EXPERIMENT 2}

\section{Detection of Orientation Changes}

The second experiment examined the ability to detect changes in the orientation of a line presented as a dynamic vibrotactile pattern. Orientation-change thresholds were determined both for dynamic patterns differing in the timing of presentation and for dynamic patterns involving length changes in addition to changes in orientation.

\section{Method}

Subjects. The subjects were 2 female and 4 male sighted introductory psychology students, who elected to participate either for course credit or for payment of $\$ 4$ per hour. No subject had previously participated in experiments on vibrotactile psychophysics (including Experiment 1) or had had prior experience using the Optacon.

Apparatus. The apparatus used was the same as that used in Experiment 1.

Stimuli. The stimuli were, once again, the recurring cycles of change in a vibrotactile line previously referred to as an event. Because no differences were found between filled and unfilled lines in the previous experiment and because of resolution constraints associated with presenting filled oblique lines at various orientations, the vibrotactile lines for the present experiment were represented by only two endpoints and a centerpoint.

The timing at which the events occurred was varied by manipulating the Ds, ISIs, and stimulus onset asynchronies (SOAs) that recurred within each event as follows: (1) D of $435.0 \mathrm{msec}$, ISI of $435.0 \mathrm{msec}$, SOA of $870.0 \mathrm{msec}$; (2) D of $435.0 \mathrm{msec}$, ISI of $217.5 \mathrm{msec}$, SOA of $652.5 \mathrm{msec}$; (3) D of $217.5 \mathrm{msec}$, ISI of $217.5 \mathrm{msec}$, SOA of $435.0 \mathrm{msec}$; and (4) D of $144.0 \mathrm{msec}$, ISI of $73.5 \mathrm{msec}$, SOA of $217.5 \mathrm{msec}$. Timing Condition 1 was identical to that used in Experiment 1. The set of timing parameters for the remaining conditions was chosen with a number of considerations in mind. First, we sought to evaluate the effects of various timing values approaching the temporal bandwidth where vibrotactile masking has previously been found, at least during letter recognition (e.g., Craig, 1976, 1983b). We also sought to maintain Ds long enough to allow for identification of the patterns, again using as a guideline data on vibrotactile letter recognition (e.g., Craig, 1983b; Loomis, 1974). Consistent with this guideline, all of the Ds used were in excess of $100 \mathrm{msec}$. Although Kirman's (1983) recent research suggests that ideal apparent motion is produced vibrotactilely with temporal overlap (i.e., negative ISIs), this overlap guarantees masking of the lines to be compared. As our primary concern was not to present ideal apparent motion, we opted to use only positive ISIs lasting no less than $73 \mathrm{msec}$. To differentiate the effects of D, ISI, and SOA, two of the conditions had the same ISI and the other two conditions had the same D. The SOAs across the four conditions were spaced at equal intervals.
The 12 orientations presented to subjects are illustrated in Figure 4. For convenience, each of the orientations has been labeled in accordance with the reference frame of the fingertip wherein a line running longitudinally represents $0^{\circ}$. Although the 12 lines shown represent the majority of lines that can be generated from the periphery of the array, the angular separation of adjacent lines, as can be noted, varies considerably. Nevertheless, because we were interested in the detection of orientation changes given the structural constraints of the vibrotactile array, we designated any change from one orientation to an adjacent orientation as a one-unit change in orientation (vtuo). The angular disparities within these units are considered in later analyses.

As in the length-change experiment, detection of movement in the dynamic pattern, rather than perception of an orientation change, would be sufficient for correct responding in the absence of any additional manipulations. To ensure that subjects were responding to perceived changes in orientation, the variable of length change was introduced. Except for orientation lines at $14^{\circ}$ and $166^{\circ}$, all lines could be represented in both a long form (L) and a short form (S). In the long form, lines were presented with their endpoints at the periphery of the array. In the short form, the lines were presented in the original orientation but at one half their original length. The outline of a rectangle within the array depicted in Figure 4 designates the location of the endpoints of lines in their short form. The first line presented in each event was either $L$ or $S$; the second line

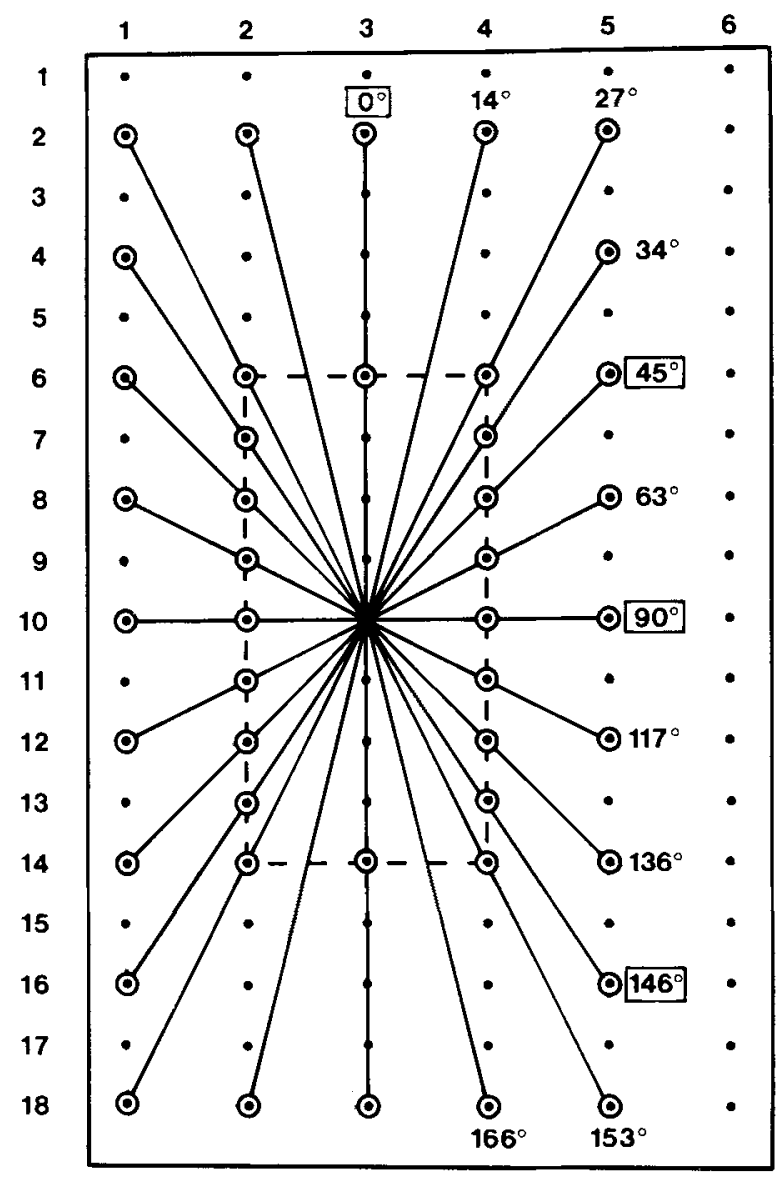

Figure 4. A representation of the 12 orientations presented. Each line consisted of two endpoints and a centerpoint. The lines were presented in a long form with endpoints at the periphery of the array or in a short form with the endpoints located as marked by the outline of the rectangle within the array. 
was independently varied between $L$ and $S$ (except for the lines at $14^{\circ}$ and $166^{\circ}$, which were always $L$ ), so that a relatively equal distribution of L-L, S-S, S-L, and L-S events resulted. Movement during an event could then be due to a change in orientation, a change in the length manipulation, or both. Figure 5 depicts a L-S event with a 2-vtuo change in orientation presented at Timing Condition 2.

It may be noted that even without this manipulation, the length of lines from one orientation to the next varies due to the shape of the Optacon array. This property of the lines complicates the analysis of the effect of changing length on the ability to detect orientation changes. This complication is addressed in later analyses, but it should be noted that the potential effects of length changes on reports of orientation changes were minimized by the explicit introduction of a length manipulation (i.e., subjects were warned that length changes were not reliable indicators of changes in orientation).

Several secondary manipulations were also introduced to assess the effects of position and direction of change on the ability to discriminate vibrotactile orientation changes. (1) The starting orientation was varied so that the first line of the event could be at an orientation of $0^{\circ}, 45^{\circ}, 90^{\circ}$, or $146^{\circ}$. These four orientations are identified in Figure 6 with enclosed line labels. (2) The direction sequence of the orientation change was varied so that movement occurred first in a clockwise then in a counterclockwise direction or first in a counterclockwise then in a clockwise direction. (3) The column position of the lines was varied so that the points of the line were displayed in columns 1,3 , and 5 or in columns 2,4 , and 6 .

Design and Procedure. The transformed up-down method described in Experiment 1 was again employed, with the magni- tude of changes in orientation determined by the subject's responses with lower and upper limits of 1 and 6 vtuo, respectively. Each block of 64 trials began with a 5-vtuo change in orientation. Where possible, the length manipulation was counterbalanced within each block, although the level remained the same for both events within a given trial. Whether the change in orientation occurred in the first or second event was determined randomly for each trial. The levels of the secondary variables of starting orientation, direction sequence, and column position were also determined randomly, with the same level presented for both events in a given trial.

In each session, two or three blocks of trials were completed in one of the four timing conditions. The timing condition received for each of the first four sessions was counterbalanced randomly for each subject, and the determined series of four timing conditions was repeated in each succeeding group of four sessions. Each subject completed as many sessions as necessary to have received at least $\mathbf{1 0 0}$ trials on either side of the $\mathbf{0 . 7 5}$ threshold for each of the four timing conditions.

The subjects were reminded at the beginning of each session to ignore length changes in the line and to attend only to changes in orientation. After each response, the next trial was presented automatically until the block was completed. The subjects were encouraged to rest between blocks and to complete only two blocks in a session if they were fatigued.

\section{Results}

As illustrated in Figure 6, the threshold for detecting changes in orientation is less than 2 vtuo for all timing

\begin{tabular}{|c|c|c|c|c|c|c|c|c|c|c|c|}
\hline & 2 & 3 & 4 & 5 & 6 & 1 & 2 & 3 & 4 & 5 & 6 \\
\hline 1 & $\cdot$ & $\cdot$ & • & $\cdot$ & $\cdot$ & $\cdot$ & $\cdot$ & $\cdot$ & $\cdot$ & $\cdot$ & $\cdot$ \\
\hline 2 & $\cdot$ & 0 & • & • & $\cdot$ & • & • & • & • & • & - \\
\hline 3 & $\cdot$ & • & - & $\cdot$ & $\cdot$ & - & • & • & • & • & • \\
\hline 4 & $\cdot$ & $\cdot$ & • & • & $\cdot$ & • & • & • & • & • & - \\
\hline 5 & • & $\cdot$ & • & • & - & • & • & • & • & • & $e^{\circ}$ \\
\hline 6 & $\cdot$ & $\cdot$ & • & • & - & - & • & $\cdot$ & ○ & • & - \\
\hline 7 & $\cdot$ & $\cdot$ & • & • & • & • & • & $\cdot$ & • & • & $\boldsymbol{\theta}^{\prime}$ \\
\hline 8 & $\cdot$ & $\cdot$ & • & • & . & • & • & $\cdot$ & • & • & - \\
\hline 9 & $\cdot$ & $\cdot$ & • & • & $\cdot$ & • & • & • & $\cdot$ & • & - \\
\hline 10 & $\cdot$ & 0 & • & • & $\cdot$ & • & • & $\circ$ & $\cdot$ & $\cdot$ & - \\
\hline 11 & $\cdot$ & $\cdot$ & • & • & $\cdot$ & • & • & • & $\cdot$ & $\cdot$ & - \\
\hline 12 & $\cdot$ & $\cdot$ & - & • & $\cdot$ & $\cdot$ & • & • & $\cdot$ & • & - \\
\hline 13 & • & $\cdot$ & - & • & $\cdot$ & • & • & • & • & $\cdot$ & • \\
\hline 14 & $\cdot$ & • & • & • & $\cdot$ & $\cdot$ & 0 & $\cdot$ & $\cdot$ & $\cdot$ & - \\
\hline 15 & $\cdot$ & • & • & • & • & • & • & $\cdot$ & • & • & - \\
\hline 16 & • & $\cdot$ & • & • & - & - & • & • & • & • & - \\
\hline 17 & • & • & • & $\cdot$ & • & - & • & • & ' & • & - \\
\hline 18 & • & 0 & - & • & - & • & • & • & - & $\cdot$ & \\
\hline
\end{tabular}

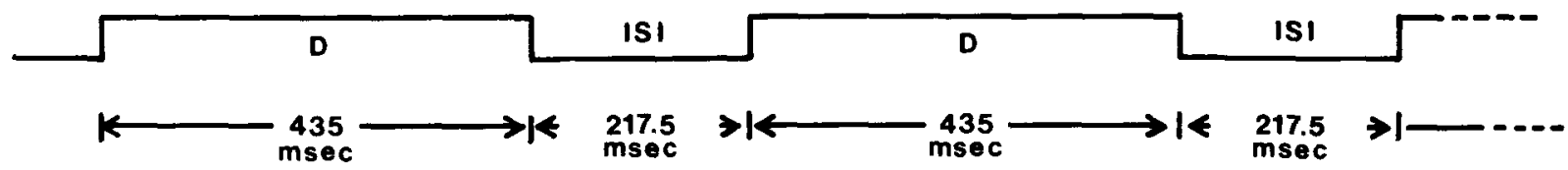

Figure 5. An example of one of the three cycles in a vibrotactile line event. The example depicts a 2-vtuo change in orientation presented at Timing Condition 2 in the L-S condition. 
conditions. In the preliminary analysis, the only manipulations significantly affecting discriminations of orientation change were length change $[F(3,5)=39.43$, $p<.001]$ and starting orientation $[F(3,5)=34.66$, $p<.001]$. The length change $\times$ starting orientation interaction was significant $[F(9,45)=7.42, p<.001]$, and the timing $\times$ starting orientation interaction just reached significance $[F(9,45)=2.11, p<.05]$. The direction sequence of orientation changes and the column position of events did not affect discriminations of orientation change. Neither did the occurrence of the orientation change in the first or second event affect performance.

The findings suggest that the timing of presentation of events within the range tested affects the discrimination of orientation very little. The timing $\times$ starting orientation interaction is difficult to interpret and does not suggest any clear advantage for a particular timing condition at any of the four starting orientations.

The interpretation of the orientation-change threshold presented and the effects of the length change and starting orientation manipulations must be approached cau- tiously. As noted earlier, the angular change is each vtuo varied somewhat across orientations. In addition, the lengths represented within the $\mathrm{L}-\mathrm{L}, \mathrm{S}-\mathrm{S}, \mathrm{S}-\mathrm{L}$, and $\mathrm{L}-\mathrm{S}$ trials varied somewhat due to the configuration of the vibrotactile array. Finally, the starting orientations differed not only in their relative position, but also in length. Rather than try to supply a weak interpretation of the above findings, we calculated the angular changes in orientation for all of the changes representing 1 and 2 vtuo. The actual lengths of the lines comprising each event were also calculated. These transformed data were then used to reanalyze the results in a multiple regression format. To retain sufficient power, the data were pooled across the timing conditions.

The first factor entered into the regression, after removal of the nonsignificant effect of direction sequence, was angular change in orientation. A log transformation of these angular changes was actually used in the analysis to bring the data into conformity with the general linear model on which regression is based. Using this measure, the angular change in orientation accounts for the first

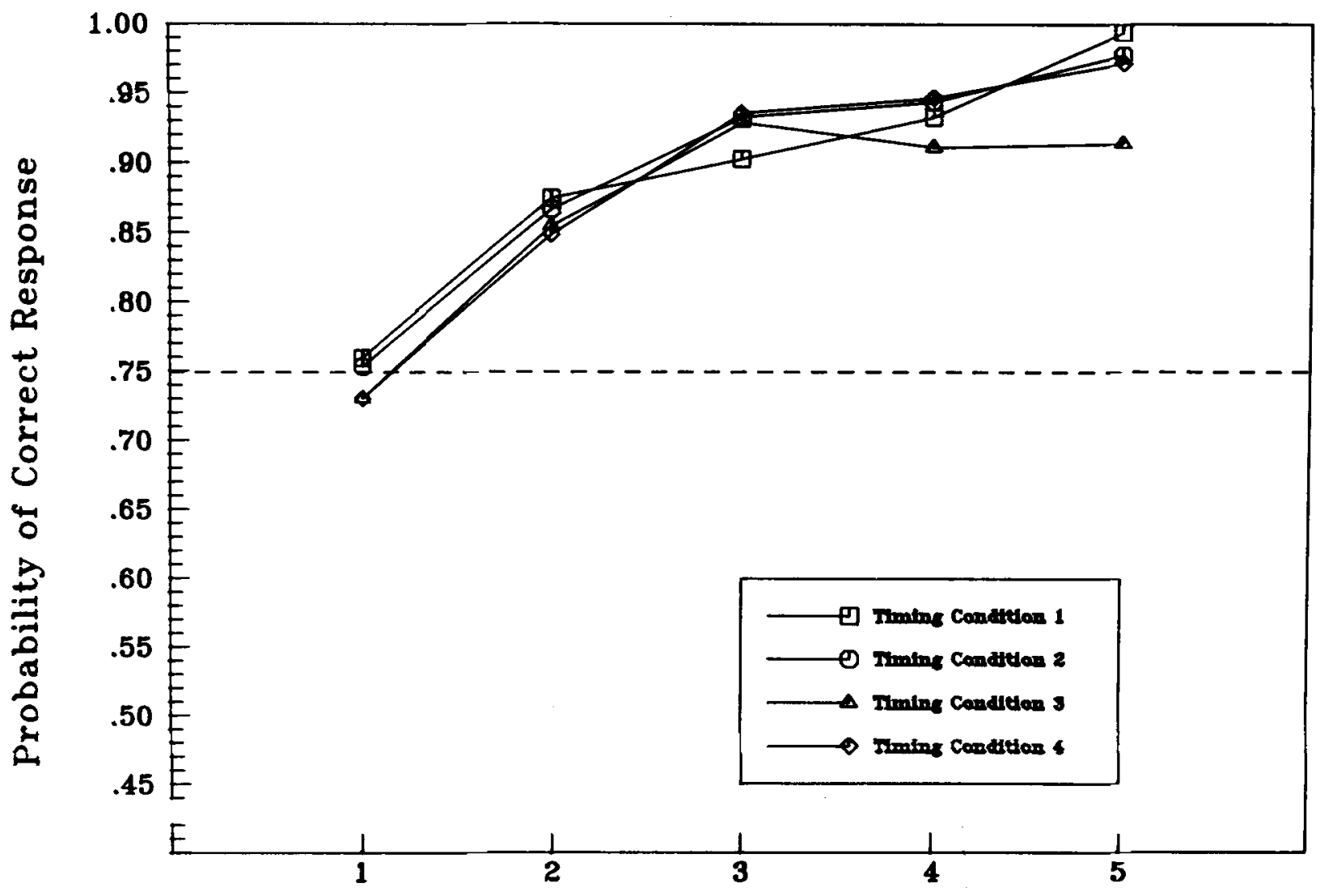

\section{Change in Orientation ( $v$ tuo)}

Figure 6. The probability of correct identification of orientation-change events as a function of the four timing conditions: (1) D of 435.0 msec, ISI of $435.0 \mathrm{msec}$, SOA of $870.0 \mathrm{msec}$. (2) D of $435.0 \mathrm{msec}$, ISI of $217.5 \mathrm{msec}$, SOA of $652.5 \mathrm{msec}$. (3) D of $217.5 \mathrm{msec}$, ISI of $217.5 \mathrm{msec}$, SOA of $435.0 \mathrm{msec}$. (4) D of $144.0 \mathrm{msec}$, ISI of $73.5 \mathrm{msec}$, SOA of $870.0 \mathrm{msec}$. The dashed line represents the 0.75 value adopted as threshold. 
$37 \%$ of the variance in subjects' performance $[F(1,55)$ $=32.48, p<.001]$.

The aim of the analysis was to clarify the effects of changes in length and positions of lines on detecting changes in orientation. To assess the effect of our lengthchange manipulation, it was necessary first to remove the effects of the sequence of length changes (shortening to lengthening vs. lengthening to shortening) and the effects of the unmanipulated length changes arising from the shape of the vibrotactile array. Neither of these covariates accounted for a significant increment in variance $[F(1,54)=2.72$ for length sequence and $F(1,53)=1.53$ for unmanipulated length changes].

Manipulated length changes, on the other hand, accounted for an additional $28 \%$ of the variance in subjects' performance $[F(1,52)=50.44, p<.001]$. Figure 7 presents the performance of subjects under the conditions examined, with log approximations to threshold functions for angular changes in orientation both with and without manipulated length changes (i.e., L-S and S-L vs. L-L and S-S). Notice that in the absence of a manipulated length change, subjects are at threshold at the smallest orientation change that can be presented on the Optacon. However, when a manipulated change in length is present, the threshold increases from a change in orientation of about $7^{\circ}$ to one of approximately $25^{\circ}$. Although the presence of a length change reduces performance accuracy, the magnitude of the length change has no discernible effect $(F<1)$.

As anticipated from our primary analysis, a significant proportion of variance $(5 \%)$ is accounted for by starting orientation $[F(1,50)=11.55, p<.01]$. It is easiest for subjects to detect changes in orientation when the starting orientation is $0^{\circ}$ and next easiest when it is $90^{\circ}$. Detection is not as accurate at $45^{\circ}$ and is even less accurate at $146^{\circ}$. This can be verified in Figures 7 and 8.

Because the remaining significant effects are relatively small, each accounting for only $2 \%-4 \%$ of the variance, they will be summarized briefly. Significant effects for the absolute starting and ending lengths of lines $[F(1,48)$ $=6.09, p<.05$, and $F(1,47)=5.99, p<.05$, respectively] suggest that subjects are more easily able to discriminate orientation changes when the lines are relatively long. The angular change in orientation $\times$ manipulated

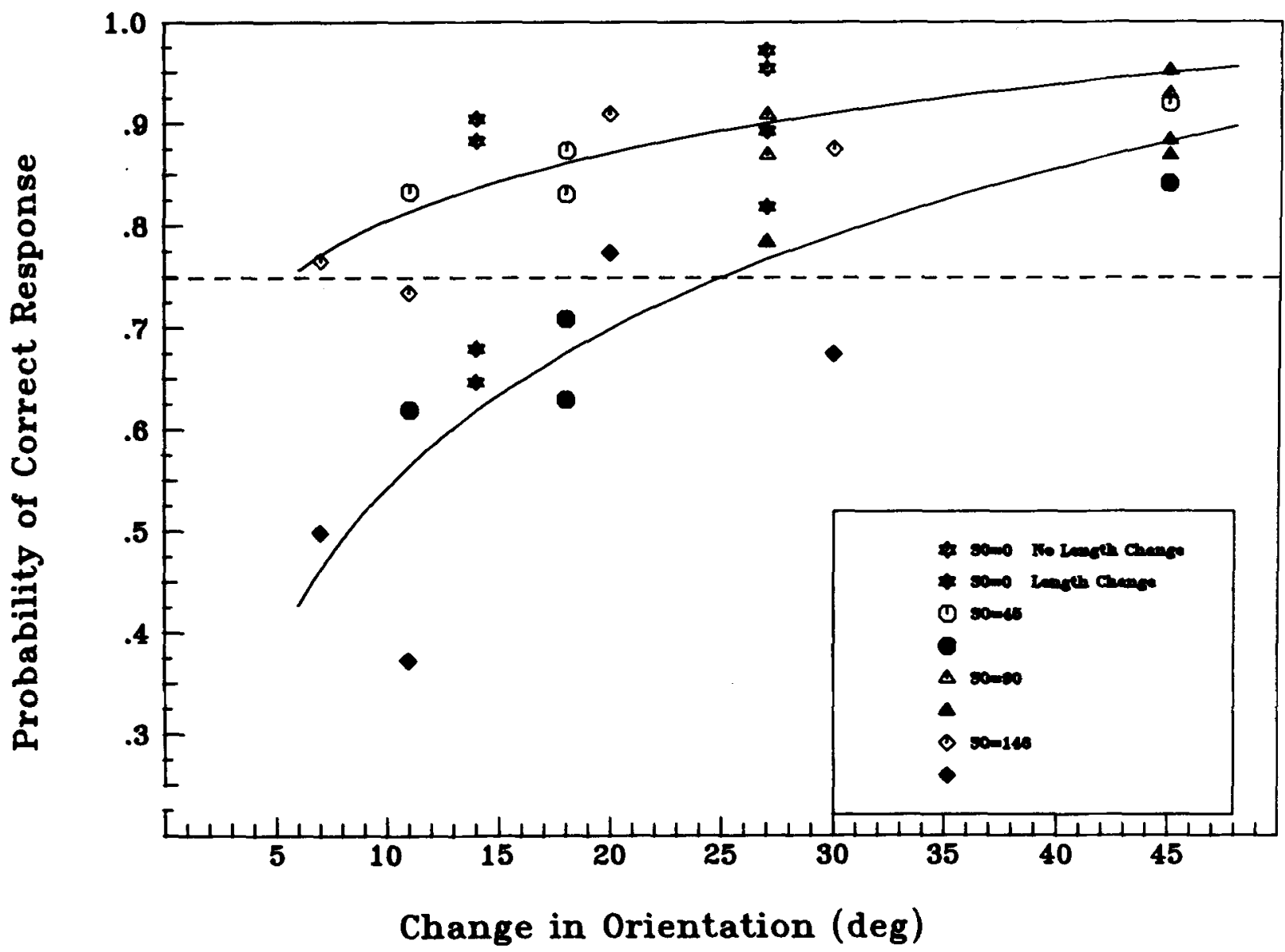

Figure 7. Log approximations to the vibrotactile orientation thresholds in the presence or absence of manipulated length changes for the angular orientation changes represented by 1 and 2 vtuo. Points marked on the graph represent the performance of subjects for the orientation changes presented given each of the starting orientations (SO). 


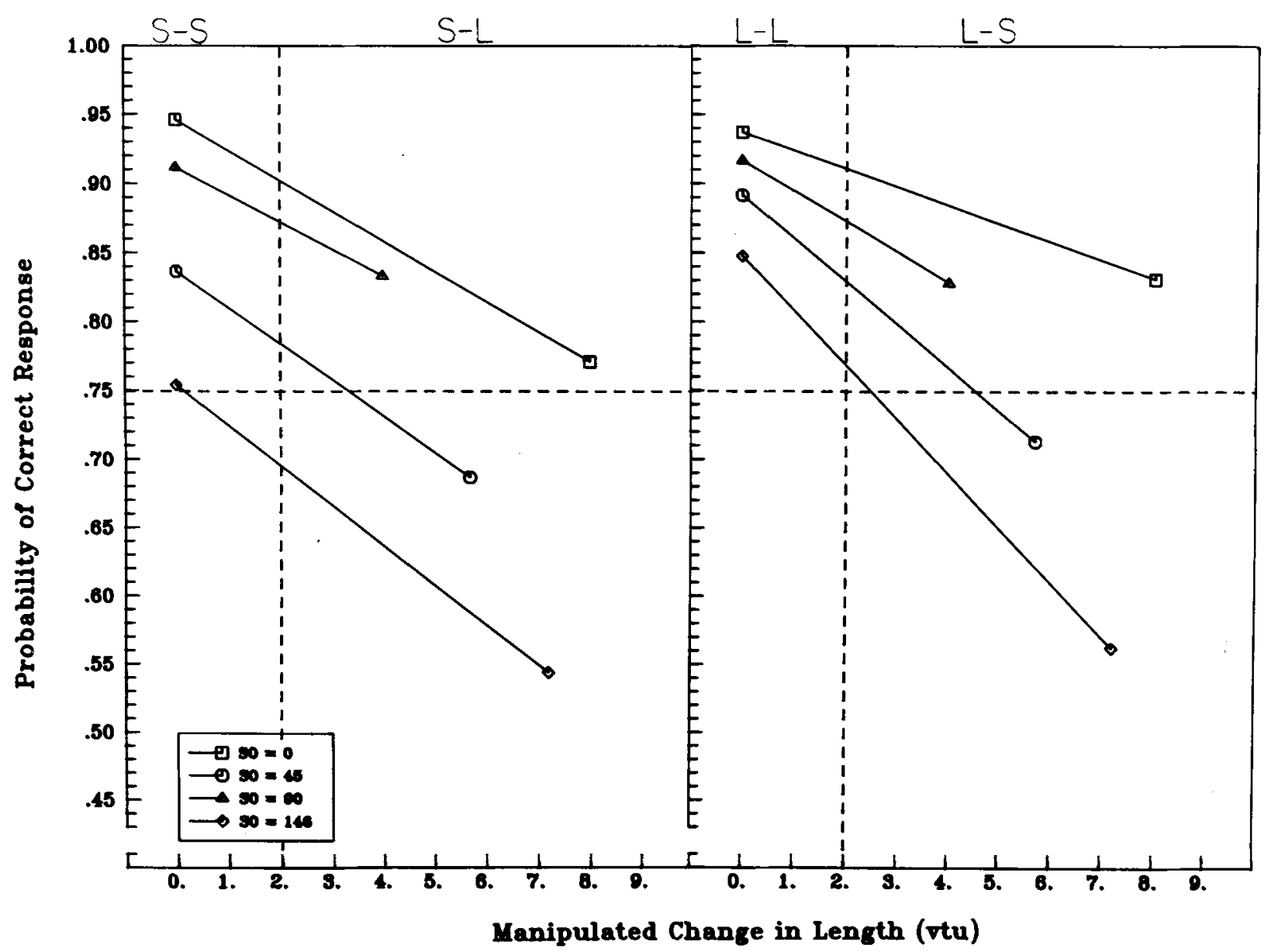

Figure 8. The probability of correct identification of orientation-change events as a function of manipulated length change, starting length, and starting orientation (SO). The dashed lines running vertically separate data from the S-S, S-L, L-L, and L-S conditions; the dashed line running horizontally represents the 0.75 value adopted as threshold.

length change interaction $[F(1,46)=12.64, p<.001]$ indicates that the ability to detect orientation changes increases at a faster rate when manipulated changes in length are present than when they are absent. This interaction is not particularly interesting given that subjects' ability to detect orientation changes in the absence of manipulated length changes is very high at almost all values of angular orientation change (i.e., there is a ceiling effect). Finally, the manipulated length change $x$ starting length interaction $[F(1,44)=6.53, p<.05]$ essentially regroups the original four levels of the length change manipulation. As illustrated in Figure 8, the L-L condition is the easiest in which to detect orientation changes, followed by the S-S, and then the S-L and L-S conditions. Notice that the latter three conditions appear to be much more susceptible to the effects of different starting orientations than is the L-L condition. Although the figure suggests a three-way interaction, the interaction did not reach significance $(F<1)$.

Overall, the independent variables in the regression account for almost $90 \%$ of the variance in subjects' perfor- mance. The vast majority of this is attributable to the effect of angular changes in orientation and the presence or absence of manipulated changes in length.

\section{DISCUSSION}

Changes in length and changes in orientation can be detected reliably for the smallest changes that can be presented on the Optacon $\left(1.2 \mathrm{~mm}\right.$ and $7^{\circ}$, respectively) provided there are no concurrent changes on other dimensions. When translations accompany changes in length, the length change threshold increases to about $4.0 \mathrm{~mm}$ (just over $3 \mathrm{vtu}$ ). Similarly, when changes in length accompany changes in orientation, the threshold for orientation change increases to about $25^{\circ}$ (just under 2 vtuo). These findings suggest that subjects are easily able to detect the presence of change, but are not as able to differentiate one kind of change from another.

Our purpose in introducing translation in Experiment 1 and length change in Experiment 2 was to exclude the possibility that the subjects' assignment of change might be 
guided by detection of displacement of a single point. Assignment of change on the basis of detection of vertical or lateral displacement of a single point would lead to correct assignment when length change (Experiment 1) and orientation change (Experiment 2) were presented alone. If it were assumed that sensitivity for point displacement was greater than sensitivity for length or orientation change, then performance would be best when detection of point displacement could determine performance accuracy but would decline when reliance on displacement was ruled out. This is one interpretation of the poorer performance when concurrent changes involving displacement were added to both events.

Another interpretation is that processing of the irrelevant dimension leads to response competition. If detection of displacement were automatic, that is, mandatory, the representation of displacement change when translation was present in Experiment 1 and length change was present in Experiment 2 might elicit a competing assignment of change. Since the competing response will be correct no more frequently than on half the trials on which it is elicited, the threshold will be elevated when irrelevant concurrent translations and length changes are present.

Third, the source of differences may occur exclusively within the decision stage. When change is present in both events and the task is to ignore nondiagnostic change, the subject may adopt a stricter criterion, requiring larger changes in length or orientation in order to assign a particular event to the length or orientation change category.

The initial orientation of lines also makes a difference in subjects' discrimination accuracy: Vertical and horizontal lines have an advantage over other oblique orientations. An initial interpretation of this effect might consider the spatial configuration of the vibrotactile array and the utilization of frames of reference in the determination of (changes in) line orientations, since implicit in any and all descriptions of orientation is one frame of reference or another. The data reveal that in our experiment the lines originating at the orientations $0^{\circ}$ and $90^{\circ}$ are more accurately judged to have changed orientation than those originating at oblique angles. The determination as to whether a line has changed its orientation necessitates, first, determining its original orientation. It may be that the orientations of $0^{\circ}$ and $90^{\circ}$ were more easily determined by subjects because they corresponded to the frame of reference afforded by the vibrotactile array or by the finger itself. The task for the subject then may have become one of determining whether subsequent presentations of that line were or were not aligned with that frame of reference. For lines with oblique starting orientations, the task is more difficult, since any one of 10 different oblique orientations could be presented on a trial. Thus, the task could have been one of identifying which of the 10 oblique lines was presented first and then deciding whether it remained at that particular oblique orientation. This sit- uation is analogous to the finding, in both the visual and the haptic literature, of oblique effects, in particular, to the finding that subjects have difficulty determining which diagonal line is presented but not in classifying whether or not a particular line is oblique (see, e.g., Appelle, 1972; Essock, 1980; Lasaga \& Garner, 1983; Lechelt, Eliuk, \& Tanne, 1976; Lechelt \& Verenka, 1980).

In addition, the detection of orientation changes is differentially affected by the absolute lengths of lines, with longer lines having an advantage over shorter lines. Although this finding suggests that the detection of length changes also may be affected by the absolute starting and ending lengths of lines, we cannot unambiguously assess the effect, since all lines in Experiment 1 began at 10 vtu.

There are several variables that do not appear to affect the detection of length and orientation changes. These include the columns and rows in which the stimuli are presented, the sequence of length changes, the direction of translations, and the direction of orientation changes. In addition, none of the timing components, including SOA, ISI, and D, systematically affect performance within the ranges examined. Finally, performance is not dependent on whether the lines making up an event are filled or unfilled.

The two experiments reported here represent an initial determination of the separate thresholds for length and orientation changes in simple patterns of vibrotactile stimulation. As such, the data reveal boundary conditions for the subsequent study of the ability of the vibrotactile system to communicate the changes correlated with the motion of objects in depth.

A number of issues remain to be more fully considered. Recall that our subjects were completely naive to vibrotactile stimulation and received no feedback. Prolonged practice and knowledge of results may substantially increase subjects' ability to differentiate changes on one dimension from changes on another. In addition, it would be useful to determine subjects' thresholds for the discrimination of concurrent changes on spatial dimensions. For instance, we do not know whether subjects' abilities to detect concurrent length and orientation changes are constrained by the thresholds for length change, for orientation change, or by some interaction of the two. If concurrent changes on two or more dimensions are made redundant rather than independent, difference thresholds may be lowered, as Sherrick (1985) has demonstrated with vibratory rate and intensity. It would be valuable to determine if this effect extends to other dimensions and to absolute thresholds. Finally, although we found no evidence that the timing conditions used in our experiments affected thresholds, the precise contribution of the temporal dimension to the vibrotactile perception of motion in depth also remains to be assessed. Such research is a crucial precursor to more precise evaluations of subjects' perception of concurrent length and orientation changes as specifying motion in depth. 


\section{REFERENCES}

APPELle, S. (1972). Perception and discrimination as a function of stimulus orientation: The 'oblique effect' in man and animals. Psychological Bulletin, 78, 266-278.

BACH-Y-RTTA, P. (1972). Brain mechanisms in sensory subsrition. New York: Academic Press.

Bliss, J. C., Katcher, M. H., Rogers, C, H., \& Shepard, R. P. (1970). Optical-to-tactile image conversion for the blind. IEEE Transactions on Man-Machine Systems, MMS-11, No. 1, 58-64.

Craig, J. C. (1976). Vibrotactile letter recognition: The effects of a masking stimulus. Perception \& Psychophysics, 20, 317-326.

Craig, J. C. (1980). Modes of vibrotactile pattern generation. Journal of Experimental Psychology: Human Perception \& Performance, 6 , 151-166.

CraIG, J. C. (1981). Tactile letter recognition: Pattern duration and modes of pattern generation. Perception \& Psychophysics, 30 , 540-546.

Craig, J. C. (1983a). The role of onset in the perception of sequentially presented vibrotactile patterns. Perception \& Psychophysics, 34, $421-432$.

Craig, J. C. (1983b). Some factors affecting tactile pattern recognition. International Journal of Neuroscience, 19, 47-58.

Craig, J. C., \& Sherrick, C. E. (1982). Dynamic tactile displays. In W. Schiff \& E. Foulke (Eds.), Tactual perception: A sourcebook. Cambridge: Cambridge University Press.

EPSTEIN, W. (1985). Amodal information and transmodal perception. In D. Warren \& E. Strelow (Eds.), Electronic sensing for the blind. Dordrecht, The Netherlands: Martinus-Nijhoff

Essock, E. A. (1980). The oblique effect of stimulus identification considered with respect to two classes of oblique effects. Perception, 9 , 37-46.

KirmaN, J. H. (1973). Tactile communication of speech: A review and synthesis. Psychological Bulletin, 80, 54-74.

KiRmaN, J. H. (1975). The effect of number of stimulators on the optimal interstimulus interval in tactile apparent movement. Perception \& Psychophysics, 17, 263-267.

KIRMaN, J. H. (1983). Tactile apparent movement: The effects of shape and type of motion. Perception \& Psychophysics, 34, 96-102.
Lasaga, M. I., \& Garner, W. R. (1983). Effect of line orientation on various information-processing tasks. Journal of Experimental Psychology: Human Perception \& Performance, 9. $215-225$.

Lechelt, E. C., Eliuk, J., Tanne, G. (1976). Perceptual orientational asymmetries: A compartson of visual and haptic space. Perception \& Psychophysics, 20, 463-469.

LeChelt, E. C., VerEnKa, A. (1980). Spatial anisotropy in intramodal and cross-modal judgments of stimulus orientation: The stability of the oblique effect. Perception, 9, 581-589.

LEviTT, H. (1971). Transformed up-down methods in psychoacoustics. Journal of the Acoustical Society of America, 49, 467-477.

Loomis, J. M. (1974). Tactile letter recognition under different modes of stimulus presentation. Perception \& Psychophysics, 16, 401-408.

Loomis, J. M. (1980). Interaction of display mode and character size in vibrotactile letter recognition. Bulletin of the Psychonomic Society, 16, 385-387.

Loomis, J. M. (1981). Tactile pattern perception. Perception, 10, 5-27.

Penner, M. J. (1978). Psychophysical methods and the minicomputer. In M. S. Mayzner \& T. R. Dolan (Eds.), Minicomputers in sensory and information processing research. Hillsdale, NJ: Erlbaum.

SHERRICK, C. E. (1985). A scale for rate of tactual vibration. Journal of the Acoustical Society of America, 78, 78-83.

Wallach, H., \& O'ConNELl, D. N. (1953). The kinetic depth effect. Journal of Experimental Psychology, 45, 205-214.

\section{NOTE}

1. The lower and upper limits for length changes (as well as the limits for orientation changes in the second experimen() have an effect on the transformed up-down method such that the probability of a correct response deviates slightly from 0.707 . The amount and direction of the deviation will depend on the number of trials occuming at each of the limits.

(Manuscript received May 7, 1986; revision accepted for publication August $6,1986$. 